Sección Médica / Medical

Artículos de investigación / Research paper

\title{
Variación poblacional de Rhodnius prolixus (Reduviidae: Triatominae) en Attalea butyracea (Arecaceae) en la Orinoquia colombiana
}

\author{
Population variation of Rhodnius prolixus (Reduviidae: Triatominae) in Attalea butyracea (Arecaceae) \\ in the Colombian Orinoquia region
PLUTARCO URBANO ${ }^{1}$, EDUWIN HINCAPIÉ2 ${ }^{2}$, VÍCTOR MANUEL ANGULO ${ }^{3}$, LYDA ESTEBAN $^{4}$
y LUÍS ALBERTO NÚÑEZ-AVELLANEDA ${ }^{5}$

\begin{abstract}
Resumen: El objetivo de esta investigación fue determinar la densidad poblacional de Rhodnius prolixus y de sus estadios de desarrollo, en bosques naturales de Attalea butyracea a lo largo de un periodo hidrológico, en ecosistemas de sabana inundable de la Orinoquia colombiana. Se muestrearon 120 palmas durante un año, usando trampas cebo vivo para Triatominae. Se halló que $R$. prolixus presenta alta densidad poblacional durante todo el año; que aumenta en época de bajas lluvias y disminuye en los meses de mayor precipitación. También se encontró que todos los estadios de desarrollo están presentes durante todo el año, aunque con diferencias en su representatividad, mostrando una disminución de la densidad a medida que aumenta la etapa de desarrollo. Por otra parte, se observaron altos índices de infestación, colonización y agrupamiento. Lo anterior sugiere que la especie presenta estabilidad de sus poblaciones en bosques de $A$. butyracea a pesar de la época climática, además de éxito reproductivo y, por ende, con individuos en capacidad de dispersión y colonización de otros micro hábitats en cualquier momento sin importar la condición climática.
\end{abstract}

Palabras clave: Chinches, Casanare, palmas, bosques naturales.

\begin{abstract}
The objective of this study was to determine the population density of Rhodnius prolixus and its developmental stages in natural forests of Attalea butyracea during a hydrological period in flooded savanna ecosystems of the Colombian Orinoquia region. One hundred twenty (120) palms were sampled over a period of one year using live bait traps for Triatomine bugs. Sampling showed that $R$. prolixus presents a high population density throughout the year, which increases during periods of low rainfall and decreases during the months of greatest precipitation. It was also found that all developmental stages of $R$. prolixus are presented throughout the year, although there are differences in their representation; showing decreases in density as developmental stages advance. Additionally, infestation, colonization and clustering were observed. The above suggests that the species presents population stability in $A$. butyracea forests without regard to climatic period, as well as reproductive success; thus individuals have the capacity to disperse and colonize other microhabitats at any time, regardless of climatic condition.
\end{abstract}

Key words: Bugs, Casanare, palms, natural forests.

\section{Introducción}

La variación temporal de una especie se observa a través del estudio de la fluctuación de su presencia y densidades poblacionales a través del tiempo (Morris 1960) dado que es una respuesta a cambios ambientales, disturbios de hábitat y competencias inter e intra específicas (Schowalter 2006). En ese sentido, muchos insectos son sensibles a cambios ambientales como temperatura y humedad que acompañan a los eventos de cambio de estacionalidad; sin embargo, algunas especies como las de la subfamilia Triatominae (Hemiptera: Reduviidae) mitigan la exposición de sus poblaciones a dichas condiciones adversas desarrollando respuestas adaptativas fisiológicas y etológicas en función de los distintos mi- cro hábitats y como estrategia de evasión a condiciones poco favorables de las épocas climáticas (Zeledón y Rabinovich 1981; Schowalter 2006; Schofield y Galvão 2009).

En ese contexto, las principales adaptaciones fisiológicas desarrolladas por especies de la subfamilia Triatominae consisten en una variación de los periodos ninfales, tasas de muda y de eclosión de huevos, longevidad de adultos, número de posturas y periodos de inanición (Zeledón y Rabinovich 1981). En cuanto a las adaptaciones etológicas los triatominos tienden a migrar de sus ecotopos originarios en algunas épocas climáticas adversas en búsqueda de alimento o refugio (Noireau y Dujardin 2001). Estas adaptaciones se reflejan en las características estructurales de la población durante un periodo de tiempo y son muy variables en especies asociadas

\footnotetext{
${ }^{1}$ Biólogo, M. Sc., Unitrópico, Grupo de Investigaciones Biológicas de la Orinoquia, GINBIO. Carrera 19 N³9-40 Yopal, Casanare, plurbanus@gmail.com. ${ }^{2}$ Biólogo, M. Sc., Unitrópico, Carrera 19 N 39-40 Yopal, Casanare, ehincapiep@hotmail.com. ${ }^{3}$ Médico, M. Sc., Universidad Industrial de Santander, Centro de Investigaciones en Enfermedades Tropicales, CINTROP. Sede UIS, Km 2 vía Guatiguará, Piedecuesta, Santander, Colombia. Teléfono: (57) 76563971; Fax: (57)76041196, pitorio@hotmail.com. ${ }^{4}$ Biólogo, M. Sc., Universidad Industrial de Santander, Piedecuesta, Colombia. Centro de Investigaciones en Enfermedades Tropicales, CINTROP. Sede UIS, Km 2 vía Guatiguará, Piedecuesta, Santander, Colombia, lestebanar@hotmail.com. ${ }^{5}$ Biólogo, Ph. D. Grupo de Investigación en Bioprospección y Conservación Biológica, Universidad de La Salle. Carrera 2 N ${ }^{\circ} 10-70$, Bogotá, D. C., Colombia. Teléfono: (57) 13535360.lanunez@unisalle.edu.co. Autor para correspondencia: Víctor Manuel Angulo. Universidad Industrial de Santander, Centro de Investigaciones en Enfermedades Tropicales, CINTROP. Sede UIS, Km 2 vía Guatiguará, Piedecuesta, Santander, Colombia. Teléfono: (57) 76563971; Fax: (57)76041196, pitorio@hotmail.com.
} 
a palmas (Zeledón y Rabinovich 1981), lo cual ha sido observado en Rhodnius neglectus (Lent, 1954), $R$. robustus (Larousse, 1927) y $R$. pallencens (Barber, 1932), especies que presentan diferentes densidades poblacionales dependiendo de la época climática (Pizarro y Romaña 1998; Longa y Scorza 2005; Gurgel-Gonçalves y Cuba-Cuba 2007).

Los estudios de D'Alessandro et al. (1984), GurgelGoncalves et al. (2004) y Abad-Franch et al. (2005) entre otros, han evidenciado que las palmas silvestres son ecotopos naturales de triatominos, principalmente de especies del género Rhodnius ya que les generan condiciones microclimáticas estables que permiten que las densidades poblacionales no varíen en función de la época climática. Además, las palmas, dadas sus características morfológicas y la posibilidad de anidación permanente de fauna silvestre que aprovecha la cantidad de biomasa que dichas plantas producen, generan las condiciones ideales como reservorios naturales de dichos insectos. Esto, garantiza la existencia de poblaciones estables durante todo el año aún en zonas con estacionalidad marcada (Romaña et al. 1999; Gaunt y Miles 2000; Galvao et al. 2003). En este contexto, la observación de la variación estructural de los estadios de desarrollo en función de la temporada climática es útil para determinar las épocas en las cuáles hay mayor cantidad de individuos en capacidad de dispersarse hacia otros ecotopos, incluyendo la vivienda humana (Pizarro y Romaña 1998) y adquiere una connotación epidemiológica por la capacidad del insecto de portar el parásito causal de la enfermedad de Chagas. Además, el análisis de las estructuras de edades de triatominos es muy útil para evaluar posibles ensayos de control, ya que determina los periodos de reproducción de la población (Gurgel-Gonçalves et al. 2004).

En consecuencia, el objetivo de esta investigación fue determinar la densidad poblacional y de los estadios de desarrollo de $R$. prolixus (Stal, 1859) asociados a bosques naturales de Attalea butyracea (Mutis ex L.f., Wess.Boer), a lo largo de un periodo hidrológico en ecosistemas de sabana inundable de la Orinoquia colombiana.

\section{Materiales y métodos}

Área de estudio. Los muestreos se realizaron en la finca Madrid, vereda el Viso, Municipio de Maní Casanare (4⒐'38”N y 72³9'22”O a 200 msnm) que según Romero et al. (2004) corresponde a un bosque alto denso en plano de inundación del río Unete a $200 \mathrm{msnm}$ (Fig. 1). Esta zona se caracteriza por presentar una marcada estacionalidad de régimen unimodal, con un periodo de altas lluvias de abril a octubre y otro de bajas lluvias durante los cinco meses restantes del año. La temperatura media anual es $27^{\circ} \mathrm{C}$ y la precipitación media mensual es 204,9 mm (IDEAM 2012).

Método. Los muestreos se realizaron durante 12 meses, desde septiembre de 2008 a agosto de 2009 , en los cuales se muestrearon 10 palmas de $A$. butyracea cada mes. Para seleccionar las palmas se estableció, al azar, un transecto distinto cada mes al interior del boque y utilizando el método de punto central y vecino más cercano planteado por Gentry (1982), se determinaron los individuos a examinar. La recolección de los triatominos se realizó utilizando trampas cebo vivo dispuestas en la corona de cada una de las palmas muestreadas, desde las 18:00 h hasta las 06:00 h del día siguiente cada mes, logrando un periodo de exposición de $12 \mathrm{~h}$ por palma (Angu-

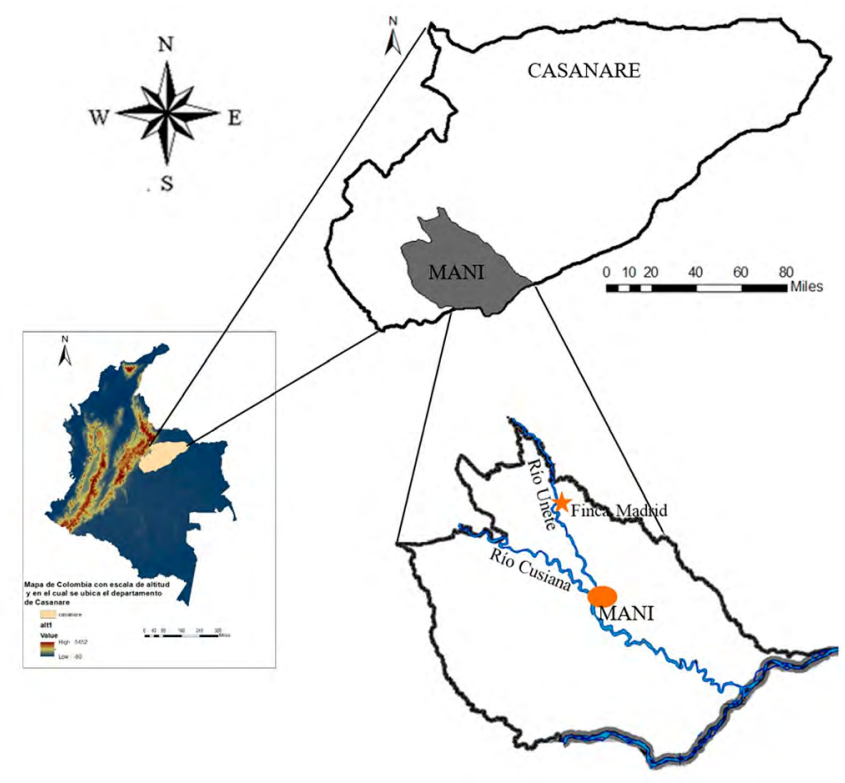

Figura 1. Ubicación geográfica de la finca Madrid en el municipio de Maní, Casanare, Colombia, donde se realizaron los muestreos para determinar la variación poblacional de Rhodnius prolixus en un bosque de Attalea butyracea. La estrella color naranja señala el punto de muestreo y el círculo color naranja está señalando el casco urbano del municipio.

lo y Esteban 2011). Se registraron la presencia y abundancias de $R$. prolixus en cada uno de los estadios de desarrollo a lo largo de los 12 meses de muestreo. La identificación taxonómica se realizó utilizando las claves de Lent y Wygodzinsky (1979) y Carcavallo et al. (1998) y también por comparación con la colección de referencia del laboratorio de entomología del Centro de Investigaciones en Enfermedades Tropicales (CINTROP) de la Universidad Industrial de Santander.

Análisis de datos. Se realizaron pruebas de Shapiro-Wilk para determinar los supuestos de normalidad de los datos, a partir del cual se realizó un análisis comparativo de las densidades registradas entre los meses muestreados utilizando Kruskal-Wallis como estadístico de prueba. Además, utilizando valores absolutos se realizó un análisis no paramétrico de comparación múltiple Dunn, para determinar la variación entre sí de la densidad poblacional por cada mes muestreado. Se realizaron gráficas de las densidades de cada estadio de desarrollo a lo largo del muestreo y del comportamiento de la densidad poblacional frente a la precipitación a lo largo de un periodo hidrológico. Por otra parte, se calcularon los índices de infestación, colonización, agrupamiento, densidad e índice adulto ninfa según los parámetros utilizados por Suárez_Dávalos et al. (2010) y Angulo et al. (2013). Para el análisis de datos se utilizó el programa estadístico Statistica 6.0 y GraphPad Prism 5.0 para las gráficas. Todos los análisis se realizaron bajo un nivel de significancia del $95 \%(\mathrm{P}<0,05)$.

\section{Resultados}

En total se recolectaron 1323 individuos identificados como Rhodnius prolixus, 246 ninfas de primer estadio (N1) (19\%), 327 de segundo (N2) (25\%), 305 de tercero (N3) (23\%), 199 de cuarto (N4) (15\%), 158 de quinto (N5) (12\%) y 88 adultos (7\%). Se observó presencia de todos los estadios de 


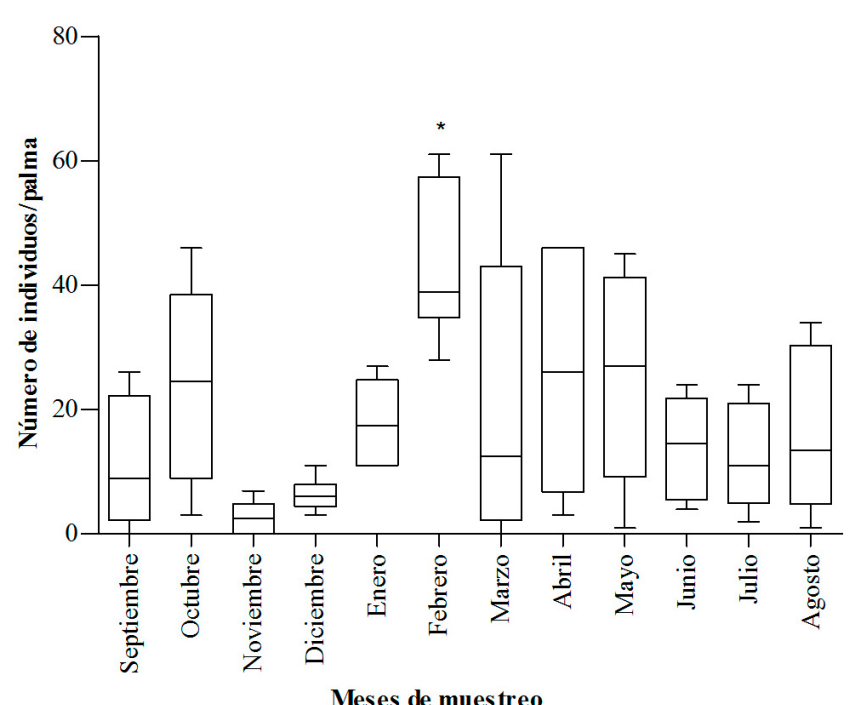

Figura 2. Variación de la densidad poblacional (número de individuos/ palma) de Rhodnius prolixus a lo largo del periodo de muestreo. Las líneas superiores e inferiores de cada caja representan los datos máximos y mínimos, respectivamente; la línea media de las cajas representa la mediana de cada uno de los grupos de datos. El asterisco (*) indica diferencias significativas $(\mathrm{P}=0,0001)$, según la prueba de Dunn. Los meses de muestreo desde septiembre a diciembre corresponden al año 2008 y desde enero a agosto al 2009.

desarrollo a largo del periodo de muestreo y según la prueba de Kruskal-Wallis $(\mathrm{P}=0,0041)$ hay diferencias significativas en las densidades recolectadas entre meses de muestreo. Según la prueba múltiple de Dunn $(\mathrm{P}=0,0001)$ febrero registró una densidad poblacional significativamente mayor frente a los demás meses (Fig. 2).

Se observaron diferencias significativas de algunos estadios de desarrollo (Kruskal_Wallis, $\mathrm{P}=0,0056$ ), como en los estadios N2 y N3 que presentan densidades mayores a los demás estadios (Dunn, $\mathrm{P}=0,0001$ ) (Fig. 3). No obstante, es importante resaltar la presencia a lo largo del periodo

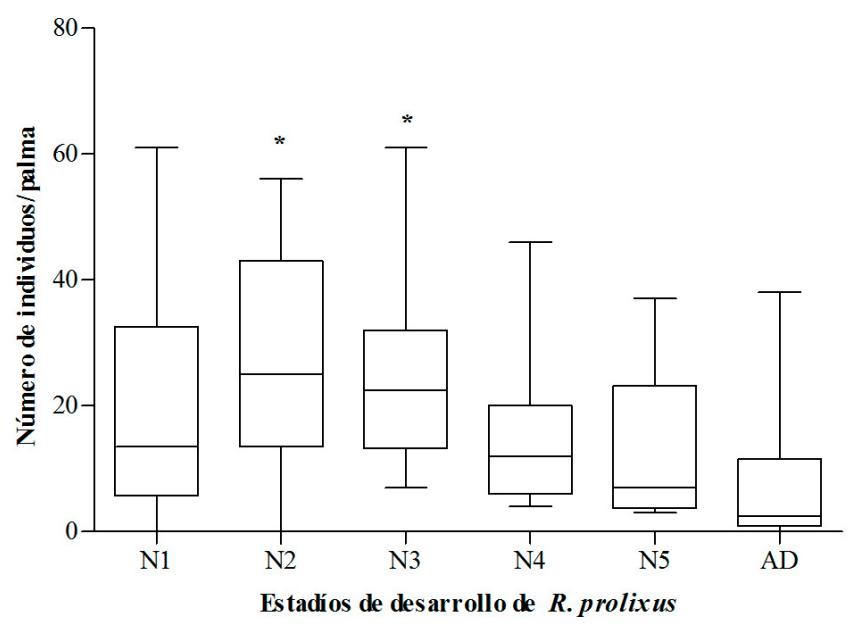

Figura 3. Densidad de los estadios de desarrollo de Rhodnius prolixus (número de individuos/palma). Las líneas superiores e inferiores de cada caja representan los datos máximos y mínimos, respectivamente; la línea media de las cajas representa la mediana de cada uno de los grupos de datos. El asterisco $(*)$ indica diferencias significativas $(P=0,0001)$, según la prueba de Dunn. Los estadios de desarrollo estan representados así: primero (N1), segundo (N2), tercero (N3), cuarto (N4), quinto (N5) y adultos $(\mathrm{AD})$. de muestreo de todos los estadios de desarrollo, ya que cada mes, por lo menos un individuo de cada estadio fue recolectado. La representatividad de los estadios de desarrollo varió a lo largo del muestreo donde los meses más disímiles fueron octubre, marzo, abril y mayo, aunque sin diferencias significativas frente a los demás (Fig. 4).

A nivel poblacional se observa que la densidad de $R$. prolixus es menor en meses que presentan mayor precipitación, con una marcada densidad poblacional en febrero el cual corresponde a uno de los meses con menor precipitación (Fig. 5). Por otra parte, se calcularon los siguientes índices: de infestación del 96,7 \%, de colonización del $98,27 \%$, de agrupamiento de 11,4 individuos por palma, de densidad de 11,0 individuos por palma y de adulto ninfa de 0,07 individuos.

\section{Discusión}

Algunas investigaciones han demostrado que la asociación entre $R$. prolixus y $A$. butyracea ocurre ya que estas palmas, regulan la variación de los cambios climáticos generando hábitat con micro-climas estables para el desarrollo de colonias de triatominos (Romaña et al. 1999; Gaunt y Miles 2000; Teixeira et al. 2001; Heger et al. 2006), lo cual podría explicar la presencia de todos los estadios de desarrollo durante todo el año (Fig. 3) y el alto índice de colonización e infestación registrado en la investigación. Además, las palmas actúan como fuente de transformación de energía en el bosque produciendo gran cantidad de flores y frutos lo que atrae a muchas especies de vertebrados que sirven como hospederos de estos insectos, lo cual le ha permitido a especies como $R$. prolixus condicionar su historia de vida a dicho micro hábitat, logrando constituir colonias estables en el tiempo y tener un éxito biológico (Romaña et al. 1999; Gaunt y Miles 2000). En ese sentido, los resultados encontrados en esta investigación sobre las altas densidades de $R$. prolixus a lo largo del muestreo (Fig. 2) y registro de todos sus estadios de desarrollo en las dos épocas climáticas (Fig. 4), nos muestran una estabilidad temporal de sus poblaciones en bosques naturales de $A$. butyracea. Por otra parte, la estabilidad temporal y la densidad tanto poblacional, como de cada uno de los estadios de desarrollo de $R$. prolixus observadas, podrían estar reflejando una eficiente utilización del micro hábitat ofertado por $A$. butyracea, dado que especies como $R$. prolixus son estrategas reproductivos tipo $\mathrm{k}$, capaces de condicionar su historia de vida a las características del micro hábitat, logrando el mantenimiento de sus colonias y el éxito biológico de la especie (Zeledón y Rabinovich 1981; Schofield y Galvão 2009), incluso en hábitats como las copas de las palmas que a pesar de presentar ambientes estables, logran una rápida saturación de su capacidad de carga (Schofield y Galvão 2009).

Particularmente en triatominos se ha observado que especies como $R$. neglectus y $R$. robustus que viven en palmas de Mauritia flexuosa (L. f., 1782) presentan mayores densidades en periodos de bajas lluvias (Gurgel-Gonçalves et al. 2004; Longa y Scorza 2005). Nuestras observaciones muestran datos similares dado que, aunque la representatividad fue similar tanto en el periodo de bajas como de altas lluvias, febrero presentó densidades significativamente mayores a las recolectadas en los demás meses de muestreo (Fig. 2). Por otra parte, la disminución marcada de la densidad poblacional en los meses de altas lluvias y un aumento leve en los meses de bajas lluvias como diciembre, enero y febrero (Fig. 5) puede 


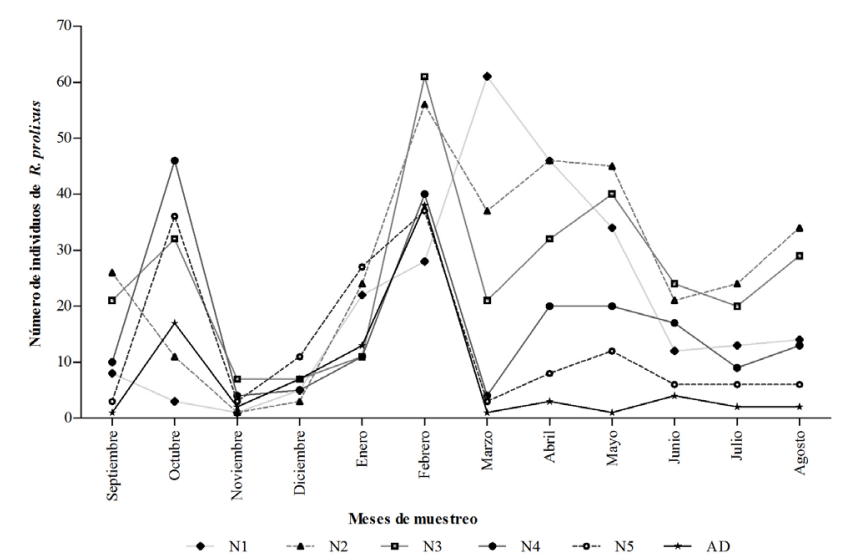

Figura 4. Número total de individuos de Rhodnius prolixus recolectado por cada estadio de desarrollo a lo largo del periodo de muestreo. Los estadios de desarrollo están representados así: primero (N1), segundo (N2), tercero (N3), cuarto (N4), quinto (N5) y adultos (AD) y los meses de muestreo desde septiembre a diciembre corresponden al año 2008 y desde enero a agosto al 2009.

indicar que $R$. prolixus no es afectado a nivel de su presencia por la variación de las condiciones ambientales en esta zona, lo que concuerda con lo observado para $R$. pallencens que habita A. butyracea (Pizarro y Romaña 1998), pero si se afecta en cuanto a su densidad poblacional de forma inversa a los valores de precipitación de las zonas de muestreo.

En este contexto, algunas investigaciones han demostrado que los valores de temperatura y humedad en las axilas y base de las hojas de palmas son más estables que los del ambiente externo (Heger et al. 2006; Urbano et al. 2015). Esta estabilidad micro climática le permite a los triatominos desarrollar su ciclo de vida sin interrupciones dadas por la variación estacional de la zona geográfica (Gurgel-Gonçalves et al. 2004). En consecuencia, las condiciones micro climáticas que estaría generando A. butyracea en esta zona le habría evitado a $R$. prolixus, restringir o volver estacionales sus ciclos de vida y, por lo tanto, se registra la presencia de todos sus estadios de desarrollo a lo largo del periodo hidrológico, como una expresión de su éxito biológico al colonizar estos ecotopos (Fig. 4). Sin embargo, la diferencia en la representatividad de los estadios de desarrollo observada (Fig. 4), podría deberse a condiciones de baja disponibilidad de alimento en el bosque dado que se ha demostrado que en condiciones de laboratorio $R$. prolixus puede variar la duración de sus estados de desarrollo como respuestas fisiológicas a la variación de la disponibilidad de recurso (Arévalo et al. 2007). También se ha observado que a medida que avanza la metamorfosis disminuyen los porcentajes de supervivencia de los estados de desarrollo (Canale et al. 1998). Esto explicaría el declive de las abundancias a partir del segundo estadio hasta la fase adulta (Fig. 3). Por otra parte, la disminución de individuos adultos puede deberse a la alta capacidad de dispersión que presenta $R$. prolixus, ligada a una alta adaptabilidad para invadir y colonizar distintos micro hábitats en áreas particulares (Feliciangeli et al. 2007) (Figs. 3-4).

Por su parte, los altos valores de índices entomológicos encontrados en colonización e infestación, muestran una estrategia importante para aumentar la probabilidad de alimentación teniendo en cuenta que el recurso alimenticio

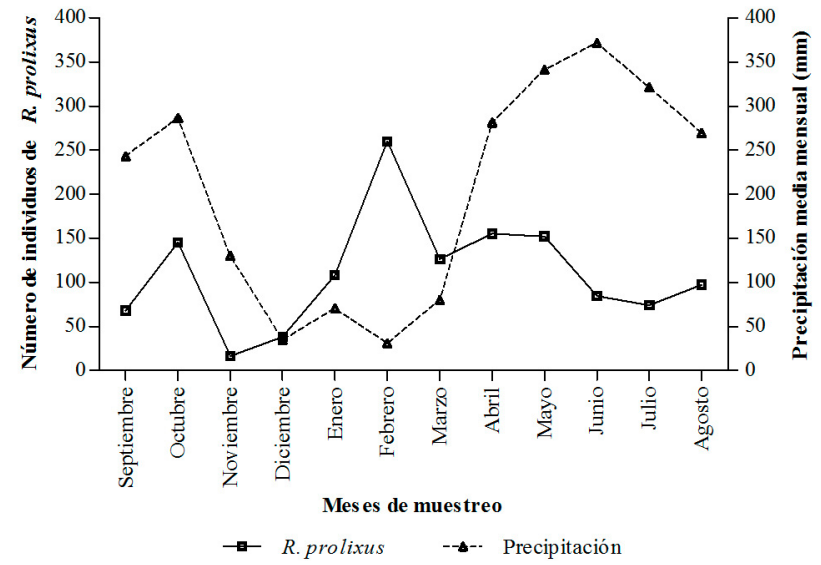

Figura 5. Variación de la poblacional de Rhodnius prolixus y la precipitación media mensual en la zona de muestreo. La línea seguida representa la densidad de $R$. prolixus registrada en el muestreo mientras la punteada los promedios mensuales de precipitación en milímetros del periodo 2007- 2008, estación Tamarindo, Casanare (IDEAM 2012). Los meses de muestreo de $R$. prolixus desde septiembre a diciembre corresponden al año 2008 y desde enero a agosto corresponden al 2009.

es móvil y, por lo tanto, ocupar todos los espacios posibles dentro del bosque, aseguraría que cualquier evento de llegada de fauna se convierta en una posibilidad de alimentación, principalmente para los estados ninfales que tienen desplazamientos restringido; no obstante dicha condición estaría siendo superada con la disposición de todos sus estadios de desarrollo en la mayoría de los microhábitats infestados, lo cual se observa en los índices de colonización y agrupamiento registrados.

La estabilidad temporal a nivel poblacional corrobora lo encontrado en otras investigaciones sobre $R$. prolixus, donde se ha mostrado que su capacidad de colonizar micro ambientes generados por otros microhábitats, depende de la viabilidad poblacional en sus ecotopos donde inicialmente establecen sus colonias (Gurgel-Gonçalves et al. 2004; Heger et al. 2006). Por lo tanto, a partir de los resultados, se puede inferir que $A$. butyracea estaría sirviendo de un centro de dispersión de individuos de $R$. prolixus, poniendo en evidencia la fuerte asociación del vector con esta especie de palma. Lo anterior se corrobora con la estabilidad temporal y con la presencia de todos sus estadios de desarrollo durante todo el año y demuestra que $R$. prolixus, importante vector de $T$. cruzi, tiene una relación tan compleja con las palmas de A. butyracea que cualquier evento que afecte estos ecotopos, repercute sobre sus poblaciones y por tanto en la ecología de la enfermedad de Chagas (Angulo et al. 2012; Rendón et al. 2015; Urbano et al. 2015; Erazo y Cordovéz 2016; Angulo et al. 2016).

\section{Conclusiones}

Los bosques de $A$. butyracea generan condiciones micro climáticas ideales para el establecimiento de colonias de $R$. prolixus, lo cual garantiza el éxito reproductivo y la estabilidad espacio temporal de la especie en términos de su estructura poblacional, sin afectación por la condición climática, corroborando la estrecha relación del vector con esta especie de palma. Estas observaciones, junto a su potencial capacidad de dispersión de adultos a otros hábitats como la vivienda 
humana, sugieren que estos bosques sean un factor de riesgo muy importante en la transmisión de $T$. cruzi a las personas que se establecen en áreas rurales donde la composición vegetal de los paisajes está dominada por palmas de $A$. butyracea, por tal razón es apremiante el diseño de estrategias de control de la intrusión de triatominos a las viviendas, ubicadas muy cerca o dentro de estos bosques.

\section{Agradecimientos}

Agradecimientos a COLCIENCIAS por la financiación de la investigación a través del contrato $\mathrm{N}^{\circ} 288-2007$ y a la profesora Katherine García del departamento de idiomas de Unitrópico, por el apoyo en el segundo idioma usado en el artículo.

\section{Literatura citada}

ABAD-FRANCH, F. S.; PALOMEQUE, H. M.; AGUILAR, H. M.; MILES, M. A. 2005. Field ecology of sylvatic Rhodnius populations (Heteroptera, Triatominae): risk factors for palm tree infestation in western Ecuador. Tropical Medicine and International Health 10 (12): 1258-1266.

ANGULO, V. M.; ESTEBAN, L. 2011. Nueva trampa para la captura de triatominos en hábitats silvestres y peridomésticos. Biomédica 31: 264-268.

ANGULO, V. M.; ESTEBAN, L.; LUNA, K. P. 2012. Attalea butyracea próximas a las viviendas como posible fuente de infestación domiciliaria por Rhodnius prolixus (Hemiptera: Reduviidae) en los Llanos Orientales de Colombia. Biomédica 32 (2): 277-285.

ANGULO, V. M.; ESTEBAN, L.; URBANO, P.; HINCAPIÉ, E.; NÚÑEZ, L. A. 2013. Comparación de métodos para la captura de triatominos (Hemiptera: Reduviidae) en palmas Attalea butyracea en los Llanos Orientales de Colombia. Biomédica 33: 653-659.

ANGULO, V. M.; CASTELlANOS, Y. Z.; FLÓREZ, M.; ESTEBAN, L.; PÉREZ, W.; FARFÁN, A. E.; LUNA, K. P. 2016. Human trypanosomiasis in the eastern plains of Colombia: New transmission scenario. The American Journal of Tropical Medicine and Hygiene 94 (2): 348-351.

ARÉVALO A.; CARRANZA, J. C.; GUHL, F.; CLAVIJO, J. A.; VALLEJO, G. A. 2007. Comparación del ciclo de vida de Rhodnius colombiensis Moreno, Jurberg \& Galvao (1999) y Rhodnius prolixus Stål (1859) (Hemiptera, Reduviidae, Triatominae) en condiciones de laboratorio. Biomédica 27 (Supl. 1): 119-129.

CANALE, B.; JURBERG, J.; CARCAVALLO, R.; GALVAO, C.; GALINDEZ, G. I.; MENA-SEGURA, C.; SILVA-ROCHA, B.; MARTINEZ, A. 1998. Bionomics of some species. pp. 839-890. En: Carcavallo, R. U.; Giron, I. G.; Jurberg, J.; Lent, H. Atlas of Chagas disease vectors in the Americas. Editorial Fiocruz, Rio de Janeiro, Brasil.

CARCAVALLO, R. U.; GALÍNDEZ, I. G.; JURBERG, J.; GALVAO, C.; LENT, H. 1998. Pictorial keys for tribes, genera and species of de subfamily Triatominae. pp. 107-244. En: Carcavallo, R. U.; Giron, I.G.; Jurberg, J.; Lent, H. Atlas of Chagas disease vectors in the Americas. Editorial Fiocruz, Rio de Janeiro, Brasil.

D'ALESSANDRO, A.; BARRETTO, P.; SARAVIA, N.; BARRETTO, M. 1984. Epidemiology of Trypanosoma cruzi in the oriental plains of Colombia. American Journal of Tropical Medicine and Hygiene 33 (6): 1084-1095.

ERAZO, D.; CORDOVÉZ, J. 2016. Modeling the effects of palmhouse proximity on the theoretical risk of Chagas disease transmission in a rural locality of the Orinoco basin, Colombia. Parasites \& Vectors 9: 592.

FELICIANGELI, D.; SANCHEZ-MARTIN, M.; MARRERO, R.; DAVIES, C.; DUJARDIN, J. P. 2007. Morphometric eviden- ce for a possible role of Rhodnius prolixus from palm trees in house re-infestation in the State of Barinas (Venezuela). Acta Tropica 101: 169-177.

GALVAO, C.; CARCAVALLO, R.; SILVA, D. R.; JURBEG, J. 2003. A checklist of the current valid species of the subfamily Triatominae Jeannel, 1919 (Hemiptera, Reduviidae) and their geographical distribution, with nomenclatural and taxonomic notes. Zootaxa 202: 1-36.

GAUNT, M.; MILES, M. 2000. The ecotopes and evolution of triatomine bugs (Triatominae) and their associated trypanosomes. Memorias do Instituto Oswaldo Cruz 95 (4): 557-565.

GENTRY, A. H. 1982. Patterns of neotropical plan diversity. Evolutionary Biology 15: 1-84.

GURGEL-GONÇALVES, R.; DUARTE, M. A.; DIAS, R. E.; TORRE-PALMA, A. R.; ROMAÑA, C. A.; CUBA-CUBA, C. A. 2004 Spatial distribution of Triatominae populations (Hemiptera: Reduviidae) in Mauritia flexuosa palm trees in Federal District of Brazil. Revista da Sociedade Brasileira de Medicina Tropical 37 (3): 241-247.

GURGEL-GONÇALVES, R.; CUBA-CUBA, C. 2007. Estructura de poblaciones de $R$. neglectus Lent e Psammolestes tertius Lent \& Jurberg (Hemiptera, Reduviidae) em ninhos de passaros (Furnaridae) presentes en la palmeira Mauritia flexuosa no distrito federal, Brasil. Revista Brasilera de Zoología 24 (1): 157-163.

HEGER, T.; GUERIN, P.; EUGSTER, W. 2006. Microclimatic factors influencing refugium suitability for Rhodnius prolixus. Physiological Entomology 31: 248-256.

IDEAM. 2012. Pronóstico IDEAM. Subsistema físico-biótico, estaciones meteorológicas de Casanare. Disponible en: http:// cdim.esap.edu.co/BancoMedios/Documentos\%20PDF/ eot $\% 2020$ tauramena $\% 20-\% 20$ casanare $\% 2020$ subsistema $\% 20$ f\%C3\%ADsico $\% 20$ bi $\%$ C3\%B3tico $\% 2020$ estaciones $\% 20$ meteorol\%C3\%B3gicas\%20\%289\%20pag\%20-\%2044\%20 kb\%29.pdf. [Fecha revisión: agosto 2016].

LENT, H.; WYGODZINSKY, P. 1979. Revision of the Triatominae (Hemiptera, Reduviidae), and their significance as vectors of Chagas disease. Bulletin of the American Museum of Natural History 163: 123-520.

LONGA, A.; SCORZA, J. V. 2005. Acrocomia aculeata (Palmae), hábitat silvestre de Rhodnius robustus en el Estado Trujillo, Venezuela. Parasitología Latinoamericana 60: 17-24.

MORRIS, R. F. 1960. Sampling insect populations. Annual Revision of Entomology 5: 243-264.

NOIREAU, F.; DUJARDIN, J. P. 2001. Flight and Nutritional Status of Sylvatic Triatoma sordida and Triatoma guasayana. Memorias do Instituto Oswaldo Cruz 96 (3): 385-389.

PIZARRO, J.; ROMAÑA, R. 1998. Variación estacional de una población silvestre de Rhodnius pallescens Barber 1932 (Heteroptera: Triatominae) en la costa Caribe colombiana. Boletín del Instituto Francés de Estudios Andinos 27 (2): 309-325.

RENDÓN, L. M.; GUHL, F.; CORDOVÉZ, J. M.; ERAZO, D. 2015. New scenarios of Trypanosoma cruzi transmission in the Orinoco region of Colombia. Memorias do Instituto Oswaldo Cruz 110 (3): 283-288.

ROMAÑA, C. A.; PIZARRO, J.; RODAS, E.; GUILBERT, E. 1999. Palm trees as ecological indicators of risk area for Chagas disease. Transactional Royal Society of Tropical Medicine and Hygiene 93: 394-395.

ROMERO, M.; GALINDO, G.; OTERO, J.; ARMENTERAS, D. 2004. Ecosistemas de la cuenca del Orinoco colombiano. Instituto de investigaciones de recursos biológicos Alexander von Humboldt. Bogotá. Colombia. 189-190.

SCHOWALTER, T. D. 2006. Insect ecology. An ecosystem approach (2nd edition). Academic Press, London, Reino Unido $576 \mathrm{p}$.

SCHOFIELD, C. J.; GALVÃO, C. 2009. Classification, evolution, and species groups within the Triatominae. Acta Tropica 110: 88-100. 
SUAREZ-DÁVALOS, V.; DANGLES, O.; VILLACIS, A. G.; GRIJALVA, M. J. 2010. Microdistribution of sylvatic Triatomine populations in central-coastal Ecuador. Journal of Medical Entomology 47 (1): 80-88.

TEIXEIRA, A. R. L.; MONTEIRO, A. S.; REBELO, J. M.; ARGANARAZ, E. R.; VIEIRA, D.; PIRES, D. L.; NASCIMENTO, R.; VEXENAT, C. A.; SILVA, A. R.; AULT, S. K.; COSTA, J. M. 2001. Emerging chagas disease: trophic network and cycle of transmission of Trypanosoma cruzi from palm trees in the Amazon. Emergy Infectious and Diseases 7 (1): 100-112.

URBANO, P.; POVEDA, C.; MOLINA, J. 2015. Effect of the physiognomy of Attalea butyracea (Arecoideae) on population density and age distribution of Rhodnius prolixus (Triatominae). Parasites \& Vectors 8: 199.
ZELEDÓN, R.; RABINOVICH, J. E. 1981. Chagas disease: And ecological appraisal with especial emphasis on its insect vectors. Annual Review of Entomology 26: 101-133.

Recibido: 18-jun-2017• Aceptado: 6-ago-2018

Citación sugerida:

URBANO, P.; HINCAPIÉ, E.; ANGULO, V. M.; ESTEBAN, L.; NÚÑEZ-AVELLANEDA, L. A. 2017. Variación poblacional de Rhodnius prolixus (Reduviidae: Triatominae) en Attalea butyracea (Arecaceae) en la Orinoquia colombiana. Revista Colombiana de Entomología 44 (2): 211-216. Julio-Diciembre 2018. 